\title{
Design of Integral Sliding Manifolds for Multi-model Uncertain Systems via LMI
}

\author{
Fernando Castaños \\ Laboratoire des Signaux \& Systèmes \\ SUPÉLEC \\ 3 rue Joliot-Curie, 91192 \\ Gif-Sur-Yvette, France \\ Email: fcas7@yahoo.com
}

\author{
Leonid Fridman \\ Universidad Nacional Autónoma de México (UNAM) \\ Department of Control Engineering \\ Engineering Faculty. \\ C.P. 04510. México D.F. \\ Email: 1fridman@verona.fi-p.unam.mx
}

\begin{abstract}
In this note we provide a way of designing the nominal trajectory of an integral sliding mode controller for linear multi-model uncertain systems. The nominal system is chosen using a mini-max criterion for the norm of the discontinuous, feed-forward control. The continuous feed-back control is then designed using linear matrix inequalities.
\end{abstract}

\section{INTRODUCTION}

Sliding mode control [1] is a robust technique, well known for its ability to withstand external disturbances and model uncertainties satisfying the matching condition, that is, perturbations that enter the state equation at the same point as the control input. Sliding mode control has other advantages as well, like ease of implementation and reduction in the order of the state equation.

A problem with conventional sliding mode control is the so called reaching phase, i.e. an initial period of time in which the system has not yet reached the sliding manifold and it is sensitive, even to perturbations satisfying the matching condition. In order to solve this problem, an integral sliding mode design concept has been proposed [2], [3], [4], [5], [6], [7]. This note is part of a research work with the aim of extending the integral sliding mode control (ISMC) technique to the case when there are external disturbances and model uncertainties that do not satisfy the matched condition.

In very general terms, the ISMC design procedure consists in choosing a nominal trajectory for the system (i.e., in the absence of perturbations and model uncertainties) and a matrix that projects the difference between the actual and nominal trajectories. The classical ISMC imposes some restrictions on both choices, but roughly speaking they can be regarded as free parameters. The main idea is to exploit this freedom and cover a broader class of control problems.

A method for choosing the projection matrix in such a way that the effect of the unmatched disturbances is minimal was presented in [8]. In this note we address the need for selecting a nominal trajectory in the presence of model uncertainties that do not necessarily satisfy the matching condition.

In this note we recognize the system matrix as a parameter which affects the norm of the equivalent control in a multimodel scenario. Furthermore, we provide an optimal value for the the worst case. We state the corresponding linear matrix inequalities (LMIs) required to take care the remaining unmatched uncertainty. We show that the order of the LMIs is reduced by the use of ISMC.

In the following section we propose an ISMC for systems having matched and unmatched disturbances but no uncertainty. In Section III we address uncertainty by using a multimodel representation and we state the problem formally. Section IV provides a criterion for selecting the nominal system trajectory. A numerical example is given in Section V. The conclusions are drawn in the last section.

\section{The Single-Model ISMC PROBLEM}

In this section we consider the problem of designing an ISMC for systems subject to external perturbations, both matched and unmatched, but without any uncertainty regarding the system's model.

\section{A. System description}

Consider a linear system of the form

$$
\dot{x}(t)=A x(t)+B u(x, t)+B_{\mathrm{w}} w(x, t),
$$

where $x \in \mathbb{R}^{n}$ is the state, $t \in \mathbb{R}$ represents time, $u(x, t) \in \mathbb{R}^{m}$ is the control action and $w(x, t) \in \mathbb{R}^{p}$ is an external disturbance. All matrices are of appropriate dimensions with $\operatorname{rank}(B)=m$. The perturbation $w(x, t)$ is of course unknown but the following is assumed:

Assumption 1: $w(x, t)$ is bounded by a known function $\bar{w}(x, t) \in \mathcal{L}_{\infty}$, i.e., $\|w(x, t)\| \leq \bar{w}(x, t)$ for all $x$ and $t$.

\section{B. Integral Sliding Mode Control}

In ISMC a feed-back control $u_{0}(x, t)$ is used to achieve the desired performance in the absence of perturbations and a feed-forward control $u_{1}(x, t)$ is used to reject the perturbations. The resulting controller then takes the form

$$
u(x, t)=u_{0}(x, t)+u_{1}(x, t) .
$$

To design the feed-forward control we start by constructing the sliding manifold $\{x \mid s(x, t)=0\}$, with

$$
s(x, t)=G\left[x(t)-x\left(t_{0}\right)-\int_{t_{0}}^{t}\left(A x(\tau)+B u_{0}(x, \tau)\right) \mathrm{d} \tau\right]
$$


and $\operatorname{rank}(G B)=m$. Then we select a gain $\rho(x, t)$, high enough such that the control

$$
u_{1}(x, t)=-\rho(x, t) \frac{(G B)^{\top} s(x, t)}{\left\|(G B)^{\top} s(x, t)\right\|}
$$

enforces a sliding motion. Note that roughly speaking, the ISMC problem is that of selecting: $G$, the feed-back control $u_{0}(x, t)$ and the gain $\rho(x, t)$.

How to choose $G$ becomes clear by first projecting the perturbation into the matched and unmatched spaces and then analyzing the system's dynamics at the sliding manifold. Consider the identity

$$
I_{n} \equiv B B^{+}+B^{\perp} B^{\perp+},
$$

where $B^{+}$is understood as the left inverse of $B$, that is, $B^{+} \triangleq\left(B^{\top} B\right)^{-1} B^{\top}$ and the columns of $B^{\perp} \in \mathbb{R}^{n \times(n-m)}$ span the null space of $B^{\top}$. Identity (4) allows us to write the perturbation as

$$
B_{\mathrm{w}} w=B B^{+} B_{\mathrm{w}} w+B^{\perp} B^{\perp+} B_{\mathrm{w}} w,
$$

so we can apply the equivalent control method as follows

$$
\begin{aligned}
\dot{s}= & G\left[A x+B\left(u_{0}+u_{1}\right)+B B^{+} B_{\mathrm{w}} w+\right. \\
& \left.+B^{\perp} B^{\perp+} B_{\mathrm{w}} w-A x-B u_{0}\right] \\
= & G B\left(u_{1}+B^{+} B_{\mathrm{w}} w\right)+G B^{\perp} B^{\perp+} B_{\mathrm{w}} w \\
u_{\mathrm{eq}}= & -B^{+} B_{\mathrm{w}} w-(G B)^{-1} G B^{\perp} B^{\perp+} B_{\mathrm{w}} w .
\end{aligned}
$$

By substituting $u_{\text {eq }}$ into the original system (1) we obtain

$$
\dot{x}=A x+B u_{0}+\left[I-B(G B)^{-1} G\right] B^{\perp} B^{\perp+} B_{\mathrm{w}} w .
$$

Remark 1: In [8] it is shown that setting $G=B^{+}$guaranties that the euclidean norm of the residual perturbation

$$
\left\|\left[I-B(G B)^{-1} G\right] B^{\perp} B^{\perp+} B_{\mathrm{w}} w\right\|,
$$

will be minimal. Moreover, since $B^{+} B^{\perp}=0$ the residual perturbation becomes $B^{\perp} B^{\perp+} B_{\mathrm{w}}$ w, i.e. the unmatched perturbation $B^{\perp} B^{\perp+} B_{\mathrm{w}} w$ is left unchanged. This means that the unmatched perturbation can not be compensated via the feed-forward control $u_{1}$ : any attempt to compensate it would only result in amplification. The choice $G=B^{+}$has other advantages as well:

- the gain satisfying the sliding condition $\dot{s} s<0$ can be set as $\rho>\left\|B^{+} B_{\mathrm{w}} w\right\|$,

- the feed-forward control (3) is simplified to

$$
u_{1}(x, t)=-\rho \frac{s}{\|s\|} .
$$

With Remark 1 in mind, we can see that the ISMC problem reduces to that of finding the feed-back control law $u_{0}$.

\section{MulTi-MODEL ISMC PROBLEM}

We consider again system (1) but we assume that the only information we have about matrix $A$, is that it is contained in the polytope

$$
A \in \mathcal{A}=\operatorname{Co}\left\{A_{i}\right\}, \quad i \in \mathcal{I}, \quad \# \mathcal{I}=N,
$$

where the symbol \# denotes the number of elements in a set and the operator $\operatorname{Co}\{\cdot\}$ denotes convex closure, i.e.

$$
\operatorname{Co}\left\{A_{i}\right\}=\left\{\sum_{i=1}^{N} \alpha_{i} A_{i} \mid \alpha_{i} \geq 0, \sum_{i=1}^{N} \alpha_{i}=1\right\} .
$$

This representation can be used, for example, to include the case of uncertain and/or time-varying parameters.

Notice that the sliding variable defined in (2) depends on $A$, and since it's precise value is unknown, we need to replace it by a nominal one

$s(x, t)=B^{+}\left[x(t)-x\left(t_{0}\right)-\int_{t_{0}}^{t}\left(A_{\mathrm{n}} x(\tau)+B u_{0}(x, \tau)\right) \mathrm{d} \tau\right]$.

By applying again the equivalent control method together with (4) we obtain

$$
u_{\mathrm{eq}}=-B^{+} B_{\mathrm{w}} w-B^{+}\left(A-A_{\mathrm{n}}\right) x .
$$

Substitution of $u_{\mathrm{eq}}$ into (1) yields

$$
\dot{x}=A x+B\left[u_{0}-B^{+}\left(A-A_{\mathrm{n}}\right) x\right]+B^{\perp} B^{\perp+} B_{\mathrm{w}} w,
$$

which, can be written as

$$
\dot{x}=A_{\text {eq }} x+B u_{0}+B_{\mathrm{w} \mathrm{eq}} w,
$$

where

$A_{\mathrm{eq}} \triangleq B^{\perp} B^{\perp+} A+B B^{+} A_{\mathrm{n}} \quad$ and $\quad B_{\mathrm{w} \text { eq }} \triangleq B^{\perp} B^{\perp+} B_{\mathrm{w}}$.

Remark 2: The matrix $B^{\perp} B^{\perp+}$ is a projection operator whose range is a subspace of dimension $n \times(n-m)$. As a consequence, the resulting polytope $\mathcal{A}_{\text {eq }}$ has a number of vertexes less than or equal to that of the original one:

$$
\mathcal{A}_{\text {eq }}=\operatorname{Co}\left\{B^{\perp} B^{\perp+} A_{i}+B B^{+} A_{\mathrm{n}}\right\}, \quad i \in \mathcal{I}_{\text {eq }} \subseteq \mathcal{I} .
$$

\section{A. Problem Statement}

In this note we restrict the class of polytopes to those describing affine parameter dependent models:

Assumption 2: The polytope $\mathcal{A}$ is of the form

$A \in \mathcal{A}=\left\{A(p) \mid A(p)=A_{0}+\sum_{i=1}^{L} p_{i} A_{i}, p_{i} \in\left[\underline{p}_{i}, \bar{p}_{i}\right]\right\}$. Notice the new polytope can be easily expressed like (6) by setting $N=2^{L}$.

Since the gain now has to mayor the norm of $B^{+} B_{\mathrm{w}} w+$ $B^{+}\left(A-A_{\mathrm{n}}\right) x$ and this term appears also in the equivalent control, we propose to minimize $\left\|A-A_{\mathrm{n}}\right\|$ for the worst case scenario:

Problem 1: Find $A_{\mathrm{n}}^{*}$ such that

$$
A_{\mathrm{n}}^{*}=\arg \min _{A_{\mathrm{n}} \in \mathbb{R}^{n \times n}} \max _{A \in \mathcal{A}}\left\|A-A_{\mathrm{n}}\right\| .
$$

\section{MAIN RESUlts}

We proceed now to determine the nominal matrix and to calculate the feed-back control 


\section{A. Selection of the Nominal System}

In order to solve the mini-max problem we start with the simplest case.

Lemma 1: The solution to the scalar, two point version of (8) is given by the average value of both points, i.e

$$
p_{\mathrm{n}}^{*} \triangleq \arg \min _{p_{\mathrm{n}} \in \mathbb{R}} \max _{p \in[\underline{p}, \bar{p}]}\left|p-p_{\mathrm{n}}\right|=\frac{\bar{p}+\underline{p}}{2}
$$

Proof: Consider the function

$$
\begin{aligned}
h_{0}\left(\alpha, p_{\mathrm{n}}\right) & =\left|\alpha \underline{p}+(1-\alpha) \bar{p}-p_{\mathrm{n}}\right|, \quad \alpha \in[0,1] \\
& =\left|\left(\frac{1}{2}-\alpha\right)(\bar{p}-\underline{p})+\frac{\bar{p}+\underline{p}}{2}-p_{\mathrm{n}}\right|
\end{aligned}
$$

and notice that for each $p_{n}$, it's image is equal to the image of $\left|p-p_{\mathrm{n}}\right|$ when $p$ varies in $[\bar{p}, p]$. Consider now the mayorant function

$$
h_{1}\left(\alpha, p_{\mathrm{n}}\right)=\left|\frac{1}{2}-\alpha\right||\bar{p}-\underline{p}|+\left|\frac{\bar{p}+\underline{p}}{2}-p_{\mathrm{n}}\right| .
$$

The maximum of $h_{1}\left(\alpha, p_{\mathrm{n}}\right)$ with respect to $\alpha$ is given by

$$
\bar{h}_{1}\left(p_{\mathrm{n}}\right) \triangleq \max _{\alpha \in[0,1]} h_{1}\left(\alpha, p_{\mathrm{n}}\right)=\left|\frac{\bar{p}-\underline{p}}{2}\right|+\left|\frac{\bar{p}+\underline{p}}{2}-p_{\mathrm{n}}\right| \text {. }
$$

Define $p_{\mathrm{c}} \triangleq(\bar{p}+\underline{p}) / 2$ and notice that

$$
\bar{h}_{1}\left(p_{\mathrm{n}}\right)=\left|\bar{p}-p_{\mathrm{c}}\right|+\left|p_{\mathrm{c}}-p_{\mathrm{n}}\right|=\left|\underline{p}-p_{\mathrm{c}}\right|+\left|p_{\mathrm{c}}-p_{\mathrm{n}}\right| .
$$

Since $p_{\mathrm{c}} \in[\underline{p}, \bar{p}] \subset\left[\underline{p}, p_{\mathrm{n}}\right] \cup\left[p_{\mathrm{n}}, \bar{p}\right]$ we know that one of the following equalities hold:

$$
\bar{h}_{1}\left(p_{\mathrm{n}}\right)=\left\{\begin{array}{c}
\left|\bar{p}-p_{\mathrm{n}}\right| \\
\text { or } \\
\left|\underline{p}-p_{\mathrm{n}}\right|
\end{array} .\right.
$$

Since for each $p_{\mathrm{n}}$ both possibilities are contained in the image of $h_{0}\left(\alpha, p_{\mathrm{n}}\right)$ and

$$
h_{0}\left(\alpha, p_{\mathrm{n}}\right) \leq h_{1}\left(\alpha, p_{\mathrm{n}}\right) \quad \forall p_{\mathrm{n}}, \quad \alpha \in[0,1],
$$

we conclude that

$$
\max _{\alpha \in[0,1]} h_{0}\left(\alpha, p_{\mathrm{n}}\right)=\max _{\alpha \in[0,1]} h_{1}\left(\alpha, p_{\mathrm{n}}\right) .
$$

Now it is easy to verify that

$$
\arg \min _{p_{\mathrm{n}} \in \mathbb{R}}\left|\frac{\bar{p}-\underline{p}}{2}\right|+\left|p_{\mathrm{c}}-p_{\mathrm{n}}\right|=p_{\mathrm{c}} .
$$

We can now determine the nominal parameters for the optimal matrix.

Proposition 1: The parameter vector achieving the minimax criterion

$$
p_{\mathrm{n}}^{*} \triangleq \arg \min _{p_{\mathrm{n}} \in \mathbb{R}^{L}} \max _{A(p) \in \mathcal{A}}\left\|A(p)-A\left(p_{\mathrm{n}}\right)\right\|,
$$

is given by $p_{\mathrm{n}}^{*}=\left(\underline{p}_{+}+\bar{p}\right) / 2$, where $\underline{p}=\left[\begin{array}{lll}\underline{p}_{1} & \cdots & \underline{p}_{L}\end{array}\right]^{\top}$ and $\bar{p}=\left[\begin{array}{lll}\bar{p}_{1} & \cdots & \bar{p}_{L}\end{array}\right]^{\top}$.

Proof: According to the definition of a matrix norm

$$
\left\|\left(A(p)-A\left(p_{\mathrm{n}}\right)\right)\right\|=\sup _{\|x\| \neq 0} \frac{\left\|\left(A(p)-A\left(p_{\mathrm{n}}\right)\right) x\right\|}{\|x\|},
$$

but $\left(A(p)-A\left(p_{\mathrm{n}}\right)\right) x$ can be rewritten as

$$
\left(A(p)-A\left(p_{\mathrm{n}}\right)\right) x=\sum_{i=1}^{L} \tilde{p}_{i} A_{i} x=\Lambda\left[\begin{array}{c}
\tilde{p}_{1} x \\
\vdots \\
\tilde{p}_{L} x
\end{array}\right],
$$

with

$$
\tilde{p} \triangleq p-p_{\mathrm{n}} \quad \text { and } \quad \Lambda \triangleq\left[\begin{array}{lll}
A_{1} & \cdots & A_{L}
\end{array}\right] .
$$

Recall that

$$
\begin{aligned}
\lambda_{\min }^{1 / 2}\left(\Lambda^{\top} \Lambda\right)\left\|\left[\begin{array}{c}
\tilde{p}_{1} x \\
\vdots \\
\tilde{p}_{L} x
\end{array}\right]\right\| \leq\left\|\Lambda\left[\begin{array}{c}
\tilde{p}_{1} x \\
\vdots \\
\tilde{p}_{L} x
\end{array}\right]\right\| \leq \\
\leq \lambda_{\max }^{1 / 2}\left(\Lambda^{\top} \Lambda\right)\left\|\left[\begin{array}{c}
\tilde{p}_{1} x \\
\vdots \\
\tilde{p}_{L} x
\end{array}\right]\right\|
\end{aligned}
$$

and since

$$
\left\|\left[\begin{array}{c}
\tilde{p}_{1} x \\
\vdots \\
\tilde{p}_{L} x
\end{array}\right]\right\|=\|\tilde{p}\|\|x\|,
$$

we conclude that $\left\|\left(A(p)-A\left(p_{\mathrm{n}}\right)\right)\right\|=\|\Lambda\|\|\tilde{p}\|$. The latter means that

$$
\begin{aligned}
p_{\mathrm{n}}^{*} & =\arg \min _{p_{\mathrm{n}} \in \mathbb{R}^{L}} \max _{p, p_{i} \in\left[\underline{p}_{i}, \bar{p}_{i}\right]}\|\Lambda\|\|\tilde{p}\| \\
& =\arg \min _{p_{\mathrm{n}} \in \mathbb{R}^{L}} \max _{p, p_{i} \in\left[\underline{p}_{i}, \bar{p}_{i}\right]}\|\tilde{p}\| .
\end{aligned}
$$

Notice that the maximum is given by

$$
\max _{p, p_{i} \in\left[\underline{p}_{i}, \bar{p}_{i}\right]}\|\tilde{p}\|=\sum_{i=1}^{L} \max _{p_{i} \in\left[\underline{p}_{i}, \bar{p}_{i}\right]}\left|\tilde{p}_{i}\right|,
$$

and the mini-max by

$$
\begin{aligned}
p_{\mathrm{n}}^{*} & =\arg \min _{p_{\mathrm{n}} \in \mathbb{R}^{L}} \max _{p, p_{i} \in\left[\underline{p}_{i}, \bar{p}_{i}\right]}|\tilde{p}| \\
& =\sum_{i=1}^{L} \arg \min _{p_{i}} \max _{p_{i} \in\left[\underline{p}_{i}, \bar{p}_{i}\right]}\left|p_{i}-p_{\mathrm{n} i}\right| .
\end{aligned}
$$

Application of Lemma 1 completes the proof.

It is clear that the matrix $A_{\mathrm{n}}^{*}$ must be inside the polytope $\mathcal{A}$, so we set $A_{\mathrm{n}}^{*}=A\left(p^{*}\right)$.

\section{B. Calculation of the Feed-back Control}

To calculate the feed-back control we rely on LMI based design techniques. An LMI has the form

$$
F(y) \triangleq F_{0}+\sum_{i=1}^{l} y_{i} F_{i}>0,
$$

where $y \in \mathbb{R}^{l}$ is the variable and the symmetric matrices $F_{i}=F_{i}^{\top} \in \mathbb{R}^{n \times n}$ are given. A useful property of LMIs is that the solution set $\{y \mid F(y)>0\}$ is convex. As explained in [9], convexity allows to solve optimization 
problems numerically in a very efficient way. Another advantage is the possibility to cast multiple LMIs, e.g. $F^{(1)} y>$ $0, \ldots, F^{(p)} y>0$, as a single one:

$$
\operatorname{diag}\left(F^{(1)} y, \ldots, F^{(p)} y\right)>0 .
$$

It may seem that the requisite of having a linear inequality greatly restricts the number of problems that can be approached. To show that this is not the case, consider the quadratic matrix inequality

$$
A^{\top} P+P A+P B R^{-1} B^{\top} P+Q<0,
$$

where $A, B, Q=Q^{\top}, R=R^{\top}>0$ are given and $P=P^{\top}$ is the variable. It can also be expressed as the LMI

$$
\left[\begin{array}{cc}
-A^{\top} P-P A-Q & P B \\
B^{\top} P & R
\end{array}\right]>0,
$$

which could be written in the form of (10) if desired.

Since several LMIs can be represented as a single one, it is easy to combine several performance criteria and solve the control problem using the same framework. Another strength of the LMI framework is that the multi-model scenario fits naturally.

To calculate the feed-back control law we propose a slightly modified version of the now classical LMI's leading to $\mathcal{H}_{2}$ and $\mathcal{H}_{\infty}$ controllers. To simplify notation let's write the vertexes of the resulting polytope as $A_{\text {eq } i} \triangleq$ $B^{\perp} B^{\perp+} A_{i}+B B^{+} A_{\mathrm{n}}$, so that

$$
\mathcal{A}_{\mathrm{eq}}=\operatorname{Co}\left\{A_{\mathrm{eq}} i\right\}, \quad i \in \mathcal{I}_{\mathrm{eq}} .
$$

If we define a performance index $z=C x+D u_{0}$, where $C$ and $D$ are free designer parameters, then according to [10] and the previous analysis, minimizing the $\mathcal{H}_{\infty}$ norm of the transfer matrix $T_{z w}$ going from $w$ to $z$ in the worst case scenario, is equivalent to the problem described in $(11)^{1}$. Minimizing the $\mathcal{H}_{2}$ norm of $T_{z w}$ is equivalent to problem (12) and can be also found in [10]. In both cases the control gain is given by $K=Y X^{-1}$. Of course, both performance criteria could be mixed if desired. Note that since $\# \mathcal{I}_{\text {eq }} \leq \# \mathcal{I}$, the order of the LMI optimization problem can be greatly reduced.

\section{NUMERICAL EXAMPLE}

Consider the following multi-model LTI system:

$$
\left[\begin{array}{l}
\dot{x}_{1} \\
\dot{x}_{2}
\end{array}\right]=\left[\begin{array}{ll}
0 & a_{1} \\
2 & a_{2}
\end{array}\right]\left[\begin{array}{l}
x_{1} \\
x_{2}
\end{array}\right]+\left[\begin{array}{l}
0 \\
1
\end{array}\right](u+w(x, t))
$$

where

$$
a_{1} \in[0.8,1.2], \quad a_{2} \in[0,3]
$$

and the objective is to minimize the $\mathcal{H}_{2}$ norm of $T_{z w}$. We can write $A$ as

$$
A=\left[\begin{array}{ll}
0 & 0 \\
2 & 0
\end{array}\right]+a_{1}\left[\begin{array}{ll}
0 & 1 \\
0 & 0
\end{array}\right]+a_{2}\left[\begin{array}{ll}
0 & 0 \\
0 & 1
\end{array}\right] .
$$

${ }^{1}$ This LMI results from the real-bounded lemma, Schur's lemma and a change of variables.
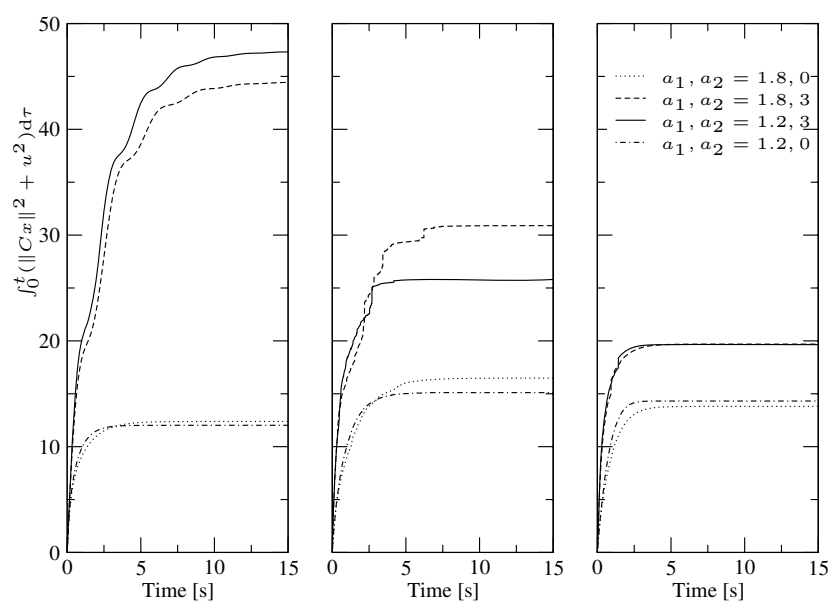

Fig. 1. Performance of the three controllers for the four different vertexes of the polytope. On the left, the LMI controller. On the center and right the LMI plus ISM controller, with $A_{\mathrm{n}} \neq A_{\mathrm{n}}^{*}$ and $A_{\mathrm{n}}=A_{\mathrm{n}}^{*}$ respectively.

With the LMI technique alone we obtain an optimal guarantied performance of $\left\|T_{z w}\right\|_{2}=9.66$. When ISMC is applied, the sliding dynamics are given by

$$
A_{\mathrm{n}}^{*}=\left[\begin{array}{cc}
0 & 1 \\
2 & 1.5
\end{array}\right] \text { and } A_{\mathrm{eq}}=\left[\begin{array}{cc}
0 & 0 \\
2 & 1.5
\end{array}\right]+a_{1}\left[\begin{array}{ll}
0 & 1 \\
0 & 0
\end{array}\right] \text {, }
$$

where we can see that the polytope now contains two vertexes instead of four. Application of the LMI technique to the new system yields $\left\|T_{z w}\right\|_{2}=4.20$.

\section{A. Simulation Results}

Three simulations were performed: the first one using the LMI technique alone; the second one using the combination LMI plus ISMC, with an $A_{\mathrm{n}} \neq A_{\mathrm{n}}^{*}$

$$
A_{\mathrm{n}}=\left[\begin{array}{ll}
0 & 0 \\
2 & 0
\end{array}\right]
$$

and the last one with $A_{\mathrm{n}}=A_{\mathrm{n}}^{*}$. Compensation of matched perturbations has been extensively simulated in the literature so we set $w(x, t)=0$, this is to highlight the issues concerning the multi-model scenario. We defined

$$
C=\left[\begin{array}{ll}
2 & 0 \\
0 & 2 \\
0 & 0
\end{array}\right] \quad \text { and } \quad D=\left[\begin{array}{l}
0 \\
0 \\
1
\end{array}\right]
$$

The initial conditions were set as $x_{1}=0.5$ and $x_{2}=1$ in all simulations. Figure 1 contains a plot of the performance

$$
\left\|z^{\prime}\right\|_{\mathcal{L} 2}^{2}=\int_{0}^{t}\left(\|C x(\tau)\|^{2}+u^{2}(\tau)\right) \mathrm{d} \tau
$$

for all the vertexes of the original polytope. Note that we have used $z^{\prime}=C x+D u$ instead of $z=C x+D u_{0}$, that is, we have included the cost of the equivalent control $u_{1}$. This is in order to establish a fair comparison between the different controllers. This also explains the fact that we have four different responses in the case of the ISMC, not just two. 
Minimize $\gamma^{2}$ over $Y, X$

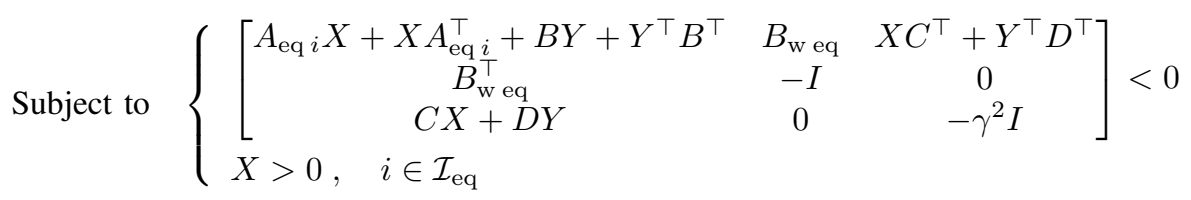

$$
\begin{array}{ll}
\text { Minimize } & \operatorname{Trace}(Q) \text { over } Y, X, Q \\
\text { Subject to }\left\{\begin{array}{cc}
{\left[\begin{array}{cc}
A_{\mathrm{eq} i} X+X A_{\mathrm{eq} i}^{\top}+B Y+Y^{\top} B^{\top} & B \\
B^{\top} & -I
\end{array}\right]<0,} \\
{\left[\begin{array}{cc}
Q & C X+D Y \\
X C^{\top}+D^{\top} Y^{\top} & X
\end{array}\right]>0, \quad i \in \mathcal{I}_{\mathrm{eq}}}
\end{array}\right.
\end{array}
$$

We can see that the performance for the worst case is improved by the use of ISMC and it is further improved when $A_{\mathrm{n}}=A_{\mathrm{n}}^{*}$.

\section{CONCLUSIONS}

We established a criteria for selecting the nominal system. It was shown that the order of the LMI's required for a multi-model case can be reduced by an ISMC. Simulations show the effectiveness of the method proposed and disprove the common miss conception that a linear system is best controlled by a linear controller.

\section{REFERENCES}

[1] V. Utkin, J. Guldner, and J. Shi, Sliding Modes in Electromechanical Systems. London, U.K.: Taylor \& Francis, 1999.

[2] G. P. Matthews and R. A. DeCarlo, "Decentralized tracking for a class of interconnected nonlinear systems using variable structure control," Automatica, vol. 24, pp. 187-93, 1988.
[3] V. Utkin and J. Shi, "Integral sliding mode in systems operating under uncertainty conditions," in Proc. Conference on Decision and Control, Kobe, Japan, Dec. 1996, pp. 4591-4596.

[4] A. Poznyak, Y. B. Shtessel, and C. Jiménez, "Mini-max sliding mode control for multimodel linear time varing systems," IEEE Trans. Automat. Contr., vol. 48, pp. 2141-2150, Dec. 2003.

[5] A. Poznyak, L. Fridman, and F. J. Bejarano, "Mini-max integral sliding mode control for multimodel linear uncertain systems," IEEE Trans. Automat. Contr., vol. 49, pp. 97-102, Jan. 2004.

[6] L. Fridman, A. Poznyak, and F. J. Bejarano, "Mini-max multimodel optimal problem via integral sliding mode control," in Proc. of the American Control Conference, Boston, June 2004, pp. 620-625.

[7] W. Cao and J. Xu, "Nonlinear integral-type sliding surface for both matched and unmatched uncertain systems," IEEE Trans. Automat. Contr., vol. 49, pp. 1355-1360, Aug. 2004.

[8] F. Castaños and L. Fridman, "Analysis and design of integral sliding manifolds for systems with unmatched perturbations," IEEE Trans. Automat. Contr., vol. 51, May 2006.

[9] S. Boyd, L. El Ghaoui, E. Feron, and V. Balakrishnan, Linear Matrix Inequalities in System and Control Theory. Philadelphia: Society for Industrial and Applied Mathematics, 1994.

[10] P. Gahinet, A. Nemierovski, A. J. Laub, and M. Chilali, LMI Control Toolbox, user guide. Natick, MA: MathWorks, Inc., 1995. 